\title{
Uniaxially Ordered Conjugated Polymer Film Prepared by Electrochemical Polymerization in a Nematic Liquid Crystal with Rubbing Orientation Method Showing Redox-Driven Tunable Dichroism
}

\author{
Kohsuke Kawabata, Hiroyuki Yoneyama and Hiromasa Goto*
}

Institute of Materials Science, Graduate School of Pure and Applied Sciences, University of Tsukuba, Tennodai 1-1-1, Tsukuba, Ibaraki, Japan. Fax:+81-298-53-4490; Tel:+81-298-53-5128; E-mail: gotoh@ims.tsukuba.ac.jp

$\dagger$ Electronic Supplementary Information (ESI) available: [details of any supplementary information available should be included here]. See DOI: 10.1039/b000000x/

We demonstrate electrochemical polymerization of an aromatic conjugated monomer in a macroscopically aligned nematic liquid crystal. This method is a combination of employment of liquid crystal for electrolyte solution, rubbing technique, and electropolymerization. The resulting polymer film shows linear dichroism and anisotropy. Polarizing optical microscopy reveals that the polymer film is uniformly ordered on a macroscopic scale. These results indicate that the conjugated main chains grow along a director of the aligned nematic liquid crystal solvent.

Recently, many researchers have studied conjugated polymers for their applications as organic electroluminescence devices, ${ }^{1}$ organic transistors, and organic photovoltaics, ${ }^{2,3}$ because of their characteristic electronic and optical properties. In this research field, the control of polymer structure represents one of the most significant current practical challenges because the molecular order of conjugated polymers can impart anisotropy and improve not only mechanical, but also electronic and optical properties. In order to obtain ordered conjugated polymers, researchers have developed many approaches, such as tensile drawing, ${ }^{4-7}$ solid-state polymerization, alignment of liquid crystalline conjugated polymers, ${ }^{8,9}$ dissolution of conjugated polymers in a liquid crystal (LC), and polymerization in a LC.

In our previous studies, electrochemical polymerization in cholesteric LC reaction fields afforded helically ordered conjugated polymer films. Electrochemical polymerization is, industrially and scientifically, one of the most useful methods for obtaining conjugated polymers because this method is straightforward and can afford a polymer thin film directly. However, generally, electrochemical polymerization can provide optically isotropic films without the possibility to orient them after polymerization. Hence, structure-controllable electrochemical polymerization is required, which can be a useful method for obtaining anisotropic conjugated polymer materials. In this 
Kohsuke Kawabata, Hiroyuki Yoneyama, and Hiromasa Goto, Polymer Chemistry (UK, RSC), 1, 1606 (2010).

research, we demonstrated that electrochemical polymerization in a macroscopically aligned nematic liquid crystal (NLC) field obtained with simple rubbing method can yield macroscopically ordered conjugated polymer films. Linearly polarized optical absorption spectroscopy and polarizing optical microscopy (POM) suggest that the polymer film is optically uniaxial on a macroscopic scale. Therefore, electrochemical polymerization in a macroscopically aligned NLC field is to be an efficient method capable of simultaneous macroscopic alignment and polymerization process.

We used 2,7-di(2-furyl)fluorene as a monomer, which was synthesised by us previously. ${ }^{10}$ The monomer was electrochemically polymerized to afford a linear aromatic conjugated polymer as shown in Scheme 1. Electrochemical polymerization in an aligned NLC reaction field was demonstrated by a following procedure. Firstly, the monomer and tetrabutyl ammonium perchlorate (TBAP) were dissolved in 4-cyano-4'-n-hexylbiphenyl (6CB). This mixture was used as an NLC electrolyte solution, in which TBAP and $6 \mathrm{CB}$ function as a supporting salt and an LC solvent, respectively. Next, rubbing treatment using poly(vinylalcohol) was conducted on surfaces of indium tin oxide (ITO) coated glass electrodes. The NLC electrolyte solution was injected between two plates of the rubbed electrodes which were separated by a $190 \mu$ m-thick Teflon spacer, and directed their rubbing to one direction. The NLC electrolyte solution between non-rubbed substrates shows a characteristic Schlieren texture (Fig. 1a), while that between rubbed substrates shows no characteristic texture due to orientation (Fig. 1b). Then, an application of a $4 \mathrm{~V}$ bias between the electrodes resulted in deposition of a polymer on the anodic electrode. After polymerization, residual NLC electrolyte solution was rinsed off with hexane to afford a green thin film. The color of the pristine polymer film is characteristic of the oxidized state due to doping of perchlorate anions for the polymer. The polymer film was reduced with hydrazine for subsequent measurements. The structure of the polymer was confirmed with IR absorption spectroscopy (Supplementary Data, Fig. S1). Film thickness is $\sim 275 \mathrm{~nm}$ (Supplementary Data, Fig. S2).

Fig. 2 shows the linearly polarized optical absorption spectra of the polymer film in the reduced and oxidized states. In these spectra, the reduced polymer exhibits an absorption band at $360 \mathrm{~nm}$ due to a $\pi-\pi^{*}$ transition of the conjugated main chain of the polymer, while the oxidized polymer exhibits two absorption bands at $360 \mathrm{~nm}$ due to a $\pi-\pi^{*}$ transition and at around $700 \mathrm{~nm}$ due to radical cations in the conjugated main chain (so-called "polarons"). In both the reduced and oxidized states, optical absorption of the polymer film with an incident polarized light parallel to the rubbing direction was more intense than in the case of the perpendicular direction. Linear dichroic ratios (Abs/// $\mathrm{Abs} \perp)$ at the maximum absorption wavelength $(360 \mathrm{~nm})$ in the reduced and oxidized states are 1.46 and 1.43 , respectively. A transition moment of $\pi$-conjugated polymers is usually along their conjugated main chain. Therefore, these results indicate that conjugated main chains in the polymer film are aligned in one direction. Interestingly, the oxidized polymer exhibits linear dichroism at the absorption band due to the polarons as well as the $\pi-\pi^{*}$ transition. This result implies that the transition moment of 
Kohsuke Kawabata, Hiroyuki Yoneyama, and Hiromasa Goto, Polymer Chemistry (UK, RSC), 1, 1606 (2010).

the polarons is also along the conjugated main chains. The optical anisotropy of the polymer film was also observable by a POM as shown in Fig. 3. Fig. 3a displays a POM photograph of the unoriented polymer. The texture is very similar to that of the NLC electrolyte solution, showing Schlieren texture. Usually electrochemically prepared polymers in isotropic solution such as acetonitrile based electrolyte solution show no Schlieren texture. This result strongly indicated that a transcription of macroscopic molecular order from NLC was occurred. While polymers prepared on rubbed substrates show no characteristic texture (Fig. 3b, c). Here, perfect aligned LCs prepared by electric or magnetic field show no characteristic texture due to complete orientation. In other words, highly oriented LCs exhibited uniform and noncharacteristic optical texture. As for the present polymer prepared in the oriented environment, the high degree of orientation led to the absence of nematic-like texture. The absence of any characteristic features of the oriented polymer strongly suggested good orientation obtained by polymerization along the oriented director field of NLC. The uniform oriented environment provides the highly oriented electro-active polymer by the transcription mechanism from the liquid crystal. Furthermore, the texture can be referred to as an ultimate form of orientation.

The polymer on the rubbed substrate under crossed Nicols showed different transmitted light intensity which depended on angles between the rubbing direction and the polarizer direction. When the angle between the rubbing direction and the polarizer direction was $45^{\circ}$, the light passed through the polymer film (Fig. 3b). On the contrary, when the angle was $0^{\circ}$, no light passed through (Fig. 3c). These results suggest that the conjugated main chain grew along a director of the macroscopically aligned NLC reaction field. Furthermore, the polymer film was uniformly aligned on a macro and mesoscopic scale. Such uniformly ordered polymer films are preferable for applications as practical electronic devices. 26

In conclusion, we have demonstrated electrosynthesis of a uniaxially ordered conjugated polymer film in a macroscopically aligned NLC reaction field. In the anisotropic NLC reaction field, electrochemical polymerization of the monomer resulted in anisotropic elongation of conjugated main chains of the corresponding polymer, which were along the director of the NLC. This anisotropic electrosynthesis with rubbing treatment is a unique, convenient, and simple method for obtaining macroscopically ordered conjugated polymer films without employment of external fields.

\section{Acknowledgement}

We would like to thank Chemical Analysis Center of University of Tsukuba and Glass Work Shop of University of Tsukuba. K. Kawabata is a research fellow of the Japan Society for the Promotion of Science. 
Kohsuke Kawabata, Hiroyuki Yoneyama, and Hiromasa Goto, Polymer Chemistry (UK, RSC), 1, 1606 (2010).

\section{References}

1 R. H. Friend, R. W. Gymer, A. B. Holmes, J. H. Burroughes, R. N. Marks, C. Taliani, D. D. C. Bradley, D. A. Dos Santos, J. L. Brédas, M. Lögdlund and W. R. Salaneck, Nature, 1997, 397, 121.

2 J. Peet, A. J. Heeger and G. C. Banzan, Acc. Chem. Res., 2009, 42, 1700.

3 O. Inganäs, F. Zhang, M. R. Andersson, Acc. Chem. Res. 2009, 42, 1731.

4 Y. Cao, P. Smith and A. J. Heeger, Polymer, 1991, 32, 1210.

5 T. W. Hagler, K. Pakbaz, K. F. Voss and A. J. Heeger, Phys. Rev. B, 1991, 44, 8652.

6 C. Weder, C. Sarwa, C. Bastiaansen and P. Smith, Adv. Mater., 1997, 9, 1035.

7 B. He, C. Wu and Y. Huang, Macromol. Chem. Phys., 2007, 208, 384.

8 I. McCulloch, M. Heeney, C. Bailey, K. Geenvicius, I. Macdonald, M. Shkunov, D. Sparrowe, S.

Tierney, R. Wagner, W. Zhang, M. L. Chabinyc, R. J. Kline, M. D. McGehee and M. F. Toney, Nature, 2006, 5, 328.

9 M. J. Banach, R. H. Friend and H. Sirringhaus, Macromolecules, 2003, 36, 2838.

10 K. Kawabata and H. Goto, Chem. Lett., 2009, 38, 706. 
Kohsuke Kawabata, Hiroyuki Yoneyama, and Hiromasa Goto, Polymer Chemistry (UK, RSC), 1, 1606 (2010).

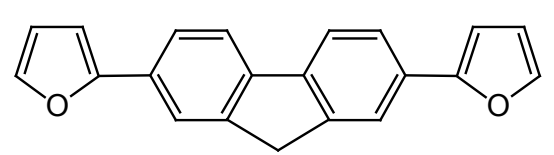

2,7-Di(2-furyl)fluorene

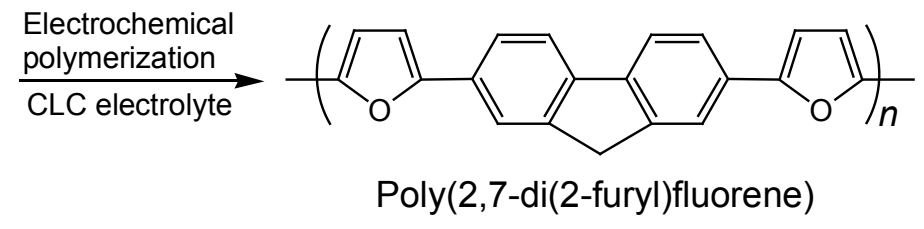

Poly(2,7-di(2-furyl)fluorene)

Scheme 1 Electrochemical polymerization of 2,7-di(2-furyl)fluorene 
Kohsuke Kawabata, Hiroyuki Yoneyama, and Hiromasa Goto, Polymer Chemistry (UK, RSC), 1, 1606 (2010).

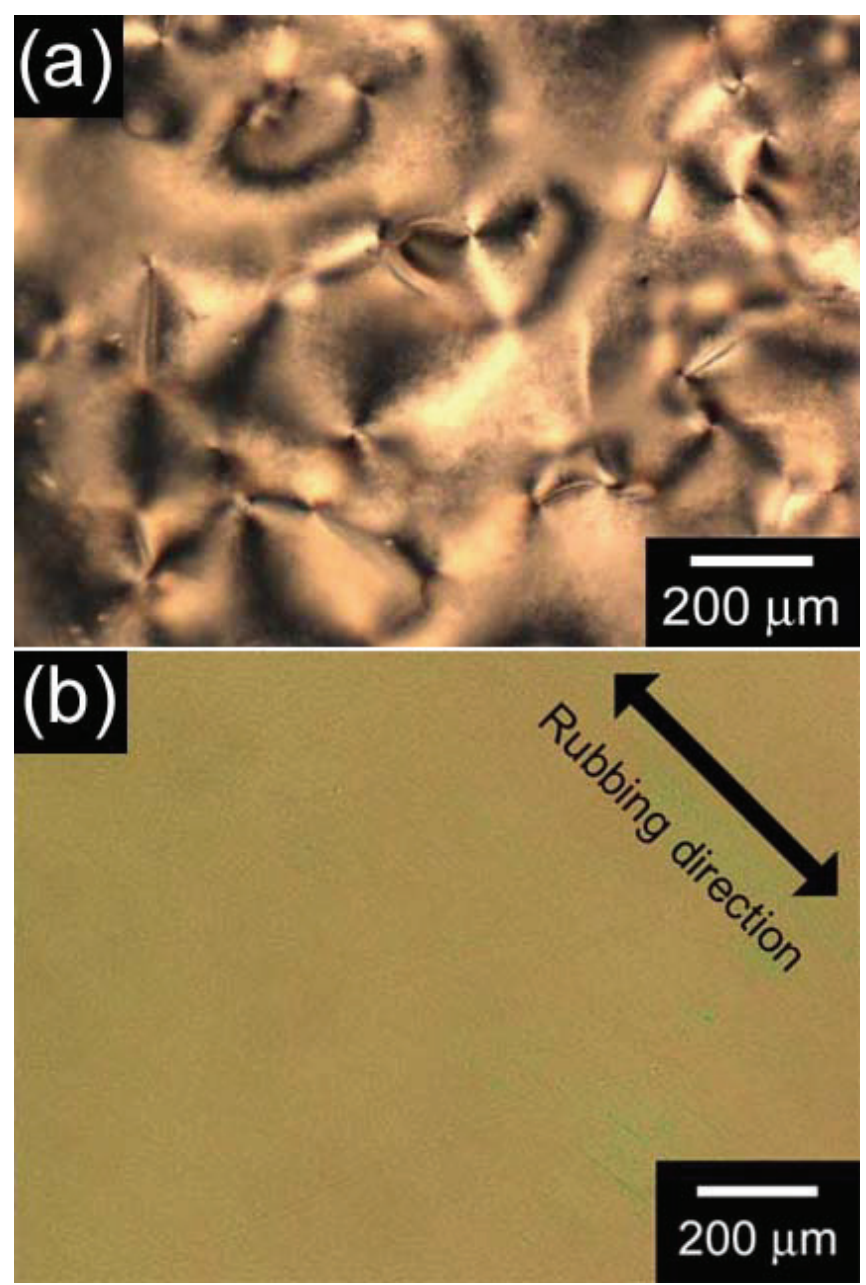

Fig. 1 Polarizing optical microscopic image of the nematic liquid crystal electrolyte solution between non-rubbed sbstrates (a), and rubbed substrates (b) 
Kohsuke Kawabata, Hiroyuki Yoneyama, and Hiromasa Goto, Polymer Chemistry (UK, RSC), 1, 1606 (2010).

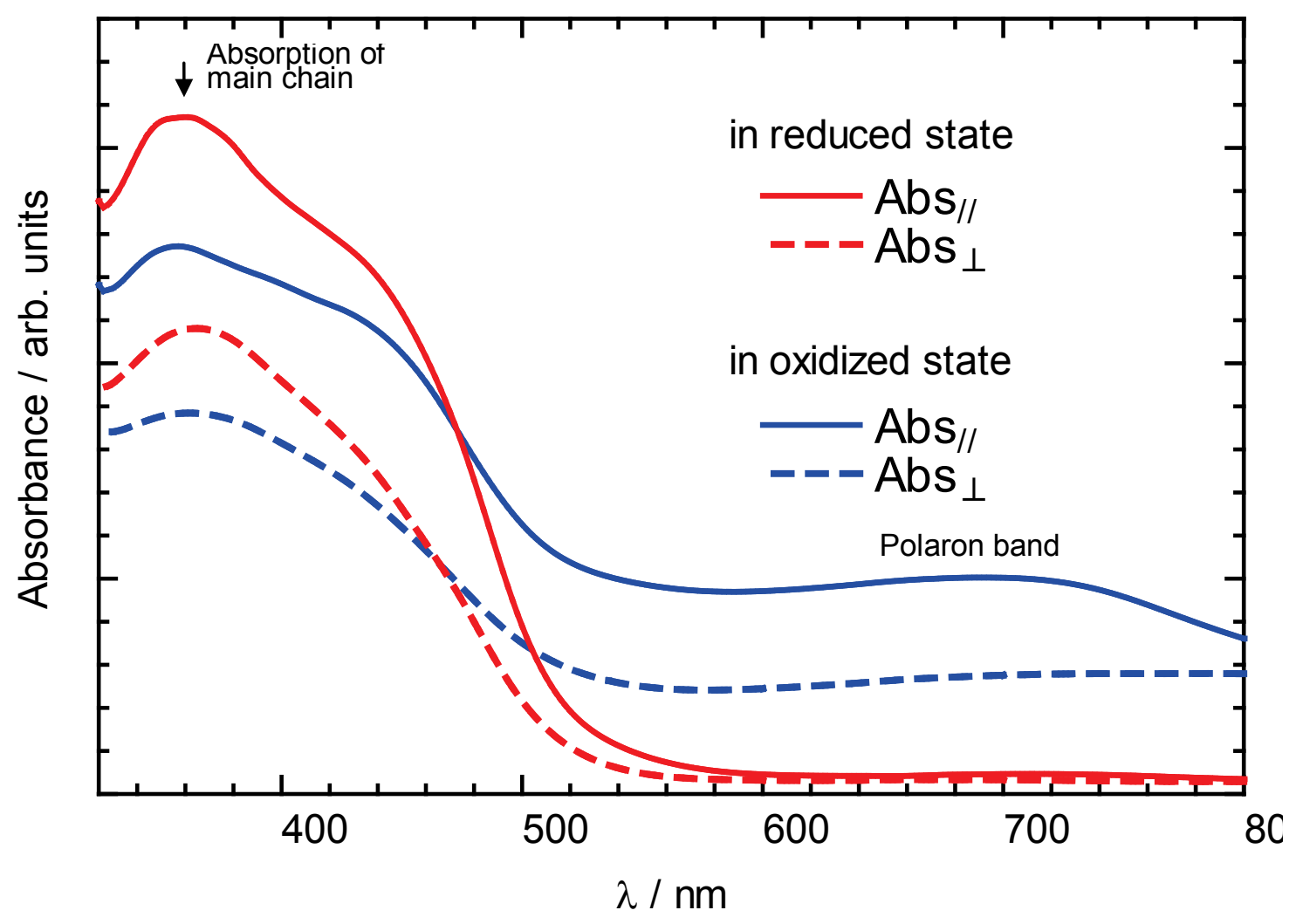

Fig. 2 Linear polarized optical absorption spectra of the polymer film in the reduced state (blue) and the oxidized state (red) with the oscillation plane of the incident polarized light parallel (solid line) and perpendicular (dashed line) to the rubbing direction 
Kohsuke Kawabata, Hiroyuki Yoneyama, and Hiromasa Goto, Polymer Chemistry (UK, RSC), 1, 1606 (2010).

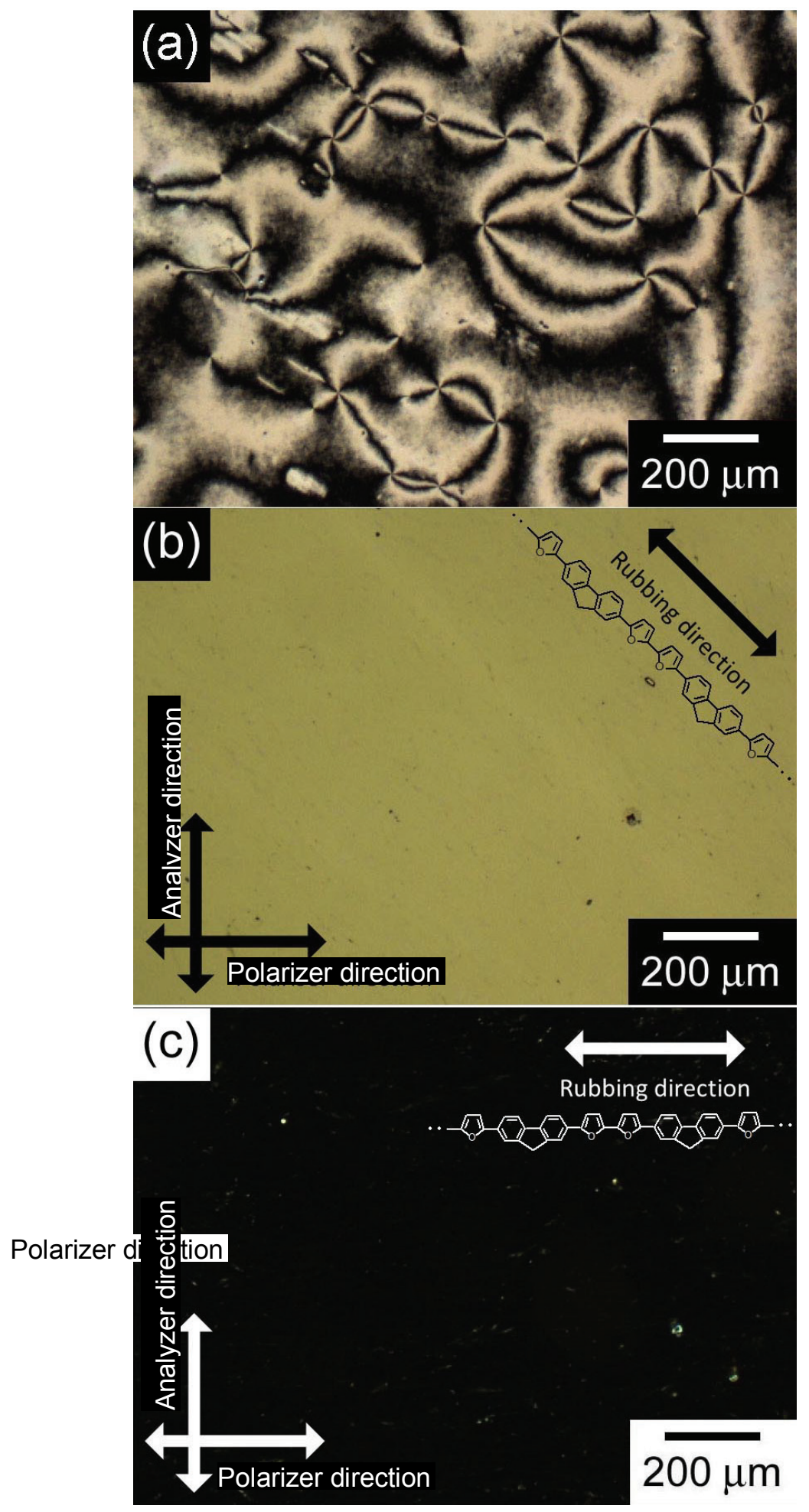

Fig. 3 Polarizing optical microscopic images of the unoriented polymer (a), and the oriented polymer film under the cross Nicol condition, in which angles between the rubbing direction and the polarizer direction are (b) $45^{\circ}$ and (c) $0^{\circ}$ 